\title{
The stable oxygen isotopic \\ composition of amorphous and \\ crystalline carbonates precipitated \\ from artificial seawater - implications \\ for nucleation and crystallisation mechanisms
}

\author{
ANTHEA I. ARNS ${ }^{1,2}$, MAXIMILIAN HANSEN ${ }^{3}$, RALF \\ SCHIEBEL $^{2}$, DENIS SCHOLZ ${ }^{3}$, ANNE JANTSCHKE ${ }^{3}$, \\ STEPHAN E. WOLF ${ }^{4}$, HUBERT VONHOF ${ }^{2}$, GERALD H. \\ HAUG $^{2,5}$ AND DAVID EVANS ${ }^{1}$ \\ ${ }^{1}$ Institute of Geosciences, Goethe University \\ ${ }^{2}$ Max Planck Institute for Chemistry \\ ${ }^{3}$ Institute of Geosciences, Johannes Gutenberg University \\ ${ }^{4}$ Friedrich-Alexander University Erlangen-Nürnberg (FAU) \\ ${ }^{5}$ ETH Zurich \\ Presenting Author: anthea.arns@mpic.de
}

Amorphous $\mathrm{CaCO}_{3}$ (ACC) is a metastable phase that is involved as a precursor in many carbonate biominerals formed by marine organisms. As such, experimental investigation of the geochemical properties of ACC precipitated under conditions relevant to marine calcifying organisms is necessary in order to improve our understanding of biomineralisation (e.g., the origin of 'vital effects'), and to provide a basis for the interpretation of stable isotope proxy data that is routinely applied in paleoclimate reconstruction.

We present $\delta^{18} \mathrm{O}$ data of ACC and crystalline carbonate precipitated via titration experiments in artificial seawater (ASW), to assess the influence of mechanisms of nucleation and crystallisation. Under the solution conditions chosen here $(\mathrm{pH}$ $\sim 9$, DIC $=\sim 20 \mathrm{mM}, \mathrm{Mg} / \mathrm{Ca}=0-5)$, the measured $\delta^{18} \mathrm{O}$ values of the precipitates implies that the dominant control of the isotope signal significantly differs for crystalline and amorphous carbonates. Relatively slow titration experiments that yield crystalline carbonates are controlled by kinetic isotope fractionation during crystal growth, as indicated by $\delta^{18} \mathrm{O}$ values lower than expected for equilibrated crystallisation. The $\delta^{18} \mathrm{O}$ values of rapidly precipitated ACC, however, are enriched in ${ }^{18} \mathrm{O}$ relative to the crystalline precipitates, resembling that calculated for $\mathrm{HCO}_{3}{ }^{-}$. This indicates that the isotopic composition of ACC is dominantly controlled by the mechanism of nucleation. For example, rapid densification during the nucleation of ACC from ASW may lead to entrapment of multiple species in a disordered structure, which means that ACC is likely to record the isotopic signal of the speciation of ions and clusters present at that time without allowing for fractionation due to the instantaneous nature of this process. Results are interpreted with respect to nonclassical nucleation and crystallisation models in order to shed light on the mechanisms of nucleation and crystallisation of ACC in seawater. 